\title{
Wireless Sensor Network based Smart Grid Communications: Challenges, protocol optimizations, and validation platforms
}

\author{
Sana Rekik • Nouha Baccour • Mohamed \\ Jmaiel · Khalil Drira
}

Received: date / Accepted: date

\begin{abstract}
Smart grids, the next generation of electric grids, require the deployment of sophisticated monitoring and control systems to enhance their operational efficiency. Wireless Sensor Networks (WSNs) have been considered as a promising communication technology for the monitoring and control of smart grid operation. They bring significant advantages such as, rapid deployment, low cost and scalability. However, the deployment of WSNs in smart grids brought new challenges mainly due to the electric grid features. Consequently, traditional WSN communication protocols have been shown inadequate and several recent research efforts were dedicated for their optimization. This paper provides a comprehensive survey on related literature, discusses the still-open research issues, and identifies the most common validation platforms for experimenting WSN communications in smart grid. We believe this survey will pave the way for the research community to (i.) understand important concepts related to WSN-based smart grid communications, (ii.) identify gaps and make valuable contributions in this timely and exiting field and (iii.) choose the convenient experimental platform for the validation of proposed solutions.
\end{abstract}

Keywords Smart grid communications · Wireless sensor networks · WSN-based smart grid communications · Protocol optimization · Validation platforms

\section{Introduction}

The need for energy conservation and environmental compliance has driven governments and utility industry over the world to transform their existing electrical grid into smart electrical grid or shortly "smart grid". Indeed, existing electrical

S. Rekik $(\bowtie) \cdot$ N. Baccour · M. Jmaiel

ReDCAD Laboratory, University of Sfax, National School of Engineers of Sfax, B.P. 1173, 3038

Sfax, Tunisia

E-mail: sana.rekik@redcad.org

K. Drira

CNRS, LAAS, 7 Avenue du Colonel Roche, F-31400 Toulouse, France 
grids have several shortcomings calling for their modernization: First, they face the challenge of limited power resources and growing power demand. Power generation in traditional electrical grids relies on power plants which mainly use fossil fuels to produce electricity. These energy resources are not only non-renewable but also environmentally unfriendly since they contribute significantly to gas emissions and climate changes. Secondly, traditional electrical grids suffer from the imbalance between power demand and supply. The amount of power to produce is generally overestimated (i.e., exceeds real power demand) in order to prevent outage in the power. This results in significant energy waste as the non-used power is dissipated due to the difficulty of its storage. Third, the legacy electrical grids lack advanced communication, monitoring and diagnostic capabilities. Electric grid utilities are not able to remotely monitor and diagnose the grid status and obtain a complete picture of the grid in real time. This is due to (i.) the limited control and sensing technologies (i.e., few wired sensors are deployed), and (ii.) the lack of modern communication networks that transfer, in real time, monitoring information from the different electric grid components to the utility.

The smart grid $[23,18]$ represents the next generation of electrical grid aiming to resolves all the above mentioned problems with the use of advanced information and communication technologies (ICT). For example, smart grids are intended to massively incorporate renewable energy sources (e.g., solar and wind) and manage their high time-variability, unpredictability and distributed generation. They are also expected to customize power demands based on the available power supplies, by carrying two-way flows of information: a flow of metering data about power consumption from the customer premises to the electric service provider and a flow of pricing information in the other direction to coerce the customers into adapting their demands. It is also foreseen that smart grids will ensure smart and real-time monitoring of the grid status through the deployment of advanced sensing capabilities.

Communication networks play an important role in the transformation of the current electric grid into smart grid $[63,25,65,32,22,43,44]$. They provide interconnections between all the grid components (e.g., generators, substations, energy storage systems, smart meters, etc.), allowing for the exchange of a large amount of information. For example, real-time information about components conditions can be exchanged for control, monitoring, and maintenance purposes. These monitoring information are of great importance as they allow avoiding the impact of electric equipment failures and natural accidents leading to power disturbances and outages.

Wireless Sensor Networks (WSNs) have been shown as a promising technology for smart grid monitoring and control applications [16,30]. They bring significant advantages compared with traditional wireless communication technologies, namely rapid deployment, large areas coverage and low cost. The potential monitoring applications enabled by WSNs in smart grids spread throughout the entire electric grid network, from power generation to transmission, distribution and consumption. For instance, in the power generation side, WSN applications include monitoring of wind farms, photo-voltaic panels and distributed energy generation $[6,45]$. As for the T\&D side, the applications of WSNs include monitoring of overhead transmission lines, underground power lines, poles and towers, and substation equipments [64,47,46,1,42]. Automatic Meter Reading (AMR), Advanced 
Metering Infrastructure (AMI) and home/building energy management are examples of WSN applications in the consumer side [21,17].

The application of WSNs in smart grids brought new challenges due to (i.) the electric grid features and (ii.) the different traffic patterns of WSN-based smart grid applications. For example, field tests conducted in [30] reveal that environmental conditions in the smart grid are complex and harsh (electromagnetic interference, obstructions, fading, etc.) turning wireless links extremely dynamic and even unpredictable. Further, WSN applications in smart grids have different quality-of-service (QoS) requirements, especially in terms of latency and reliability [32], requiring the design of differentiated communication protocols.

A fundamental question that would precede any investigation in this field is "whether existing WSN communication protocols and standards are adequate for smart grid applications?". Prior research works were dedicated for providing response elements to this question $[11,61,68]$. Several well-known technologies and protocols were evaluated such as, ZigBee, IEEE 802.15.4, IEEE 802.11, AODV, etc. As a result, all these technologies and protocols were found not sufficiently adequate for smart grid applications.

Currently, several research efforts are devoted for the optimization of existing WSN communication protocols in order to cope with the new constraints emerged from WSN-based smart grid applications. This fact motivates us to provide a survey that synthesizes and classifies the related literature and also explores the still-open research issues in this promising and timely field. To achieve this goal, we adopted the following methodology: After reviewing related work (Section 2), we first present fundamental concepts of communication networks in the smart grid, including architectures, technologies and requirements (Section 3). Then, we discuss the design challenges raised by WSNs in smart grid control and monitoring applications (Section 4). Next, we provide a taxonomy of the most recent advances in the design of new or optimized communication protocols for WSN-based smart grid communications and discuss the open issues (Section 5). Finally, we present the most common validation platforms for the performance evaluation of WSN communication protocols in smart grids (Section 6), followed by our conclusion (Section 7).

\section{Related work}

Several surveys have been introduced to tackle the different aspects of smart grid communications in general, such as the communication infrastructure [65], architecture [32], requirements [32], design challenges [53], energy efficiency [19], etc. However, to our knowledge, except [20], no previous survey paper tackled in detail WSN-based communication in smart grids. In [20], Fadel et al. surveyed the wide range of WSN applications in the different smart grid sides (i.e., generation, T\&D and consumer sides). They also discussed the adequacy of traditional WSN MAC and routing protocols (such as RAP, ReInforM, RT-MAC, QoS-MAC, etc) for smart grid by analyzing their features and capabilities and checking whether they meet smart grid constraints. The authors argued that these protocols are inherently inadequate for smart grid applications.

This paper builds on the survey of Fadel et al. [20], and provides a taxonomy of recent advances on the optimization and design of WSN communication protocols 
for smart grid applications while discussing the still-open research issues. Further, it provides a useful guide for network designers to choose the convenient validation platform for their introduced protocols.

\section{Smart grid communications}

From an architectural perspective, the smart grid consists of three main layers: energy layer, communication layer and information technology layer [25,32]. The energy layer is comprised of power generation, transmission and distribution systems, and consumer premises. The information technology layer collects and manages data coming from different components of the smart grid. The communication layer presents the core of the whole smart grid infrastructure. Communication networks support two-way flows of information between all devices in the grid (e.g., generators, substations, energy storage systems, smart meters, etc.). Exchanged information represents either (i.) energy consumption and price, allowing for a balance between energy demand and supply or (ii.) the conditions of smart grid components, allowing for their control, monitoring, and maintenance.

A variety of communication technologies can be exploited in smart grid communications, ranging from wired, such as optical fiber and Power Line Communications (PLC), to wireless technologies, such as Wireless Mesh Networks, Wireless Sensor Networks, cellular communication systems and satellite communications $[23,31,25,63,7]$. Wired technologies have the disadvantage of incurring high cost for deployment and maintenance, which limits the network scalability. Consequently, wireless communication technologies have more widespread use in smart grid networks, compared to wired technologies [34]. In particular, WSNs have been shown as a key communication technology for the control and monitoring applications in smart grids. This is due to their intrinsic characteristics, such as low cost, wide coverage and easy installation. However, WSNs cannot operate alone. They are intended to cooperate with other network technologies (e.g. high speed networks) to ensure better coverage $[64,24,69]$. For instance, the combination of WSNs and wide area networks such as cellular networks was proposed in [24], to meet the requirements of the transmission line monitoring application, namely high bandwidth and low latency.

Generally, all involved communication networks in the smart grid (including WSNs) have to cooperate together to allow the exchange of different types of data, while meeting the main communication requirements of smart grid applications, namely latency, reliability, bandwidth, security, privacy, scalability and interoperability $[31,63,65,7,38]$. These requirements are elaborated next:

- Latency: In the smart grid, most of information must be delivered within hard time bounds. When the communication delay exceeds a certain threshold, the information becomes outdated, which may lead to a damage in the grid. The International Electrotechnical Commission (IEC) and the Institute of Electrical and Electronics Engineers (IEEE) specified expected values of communication delays for different categories of exchanged information in the smart grid (e.g., maintenance information, protection information, video data streams, etc.) [63]. For example, in the circuit breaker protection application, sensed data must be received within a delay of $3 \mathrm{~ms}$ in order to make the necessary protection actions [63]. 
- Reliability: It generally refers to high end-to-end data delivery. In smart grid applications, exchanged messages have different levels of reliability: some of them may occasionally tolerate losses, while others require a high level of reliability (up to $99.99 \%$ ) [63,7]. Smart grid communication networks must allow applications to select between different levels of reliability.

- Bandwidth: The number of interconnected intelligent devices is anticipated to grow rapidly with the evolution of the smart grid. Thus, the bandwidth demands are also growing [32]. The bandwidth of smart grid communication networks must increase as fast as the demand of the connected elements in the grid to ensure low transmission latency and to reduce packet losses.

- Security and privacy: Providing communication security, especially protecting the customers' privacy, is of great importance for smart grid applications to prevent vulnerabilities to the critical assets of the electric grid. This vulnerability is the consequence of the high penetration and the variable nature of renewable energy generators, the deployment of smart meters, and the large geographical area covered by the smart grid [23,7]. Without appropriate security mechanisms and privacy protection methods, interested parties access to the electric system and destabilize the grid (e.g., altering the information stored in smart meters).

- Scalability: Smart grid communication networks have to accommodate more and more users and smart devices (e.g. smart sensors, smart meters). This raises the issue of scalability in the smart grid, which may be handled with the use of IP-based networks, web services, reliable protocols, etc [31,65].

- Interoperability: Building an interoperable smart grid communication infrastructure is a major challenge. Indeed, smart grid incorporates diverse networks, which are intended to cooperate and exchange information. The adoption of standards and open network architectures (using for example IP-based networks), while avoiding being isolated into proprietary protocols; will play a crucial role for interoperability at all the network layers $[65,7]$. IP-based standards may be considered as a promising solution to achieve interoperability in smart grid communications. For instance, WSN standards introduced by the IETF, including 6LoWPAN and RPL, can ensure high interoperability (with IPv6-based networks). In addition to the use of standardized protocols, standard interfaces and networking elements (e.g., gateways) may be introduced to provide communications between different networks that use different standards.

\section{Protocol design challenges in WSN-based smart grid applications}

WSNs have unique characteristics that make them different from any other wireless network. For example, sensor devices are designed with constrained resources in terms of energy, memory, and processing capabilities. Further, they use low cost and low-power radios, which turn communication links prone to noise, interference and multi-path effects. These features have been well considered in the design of major WSN communication protocols.

Smart grid monitoring applications represent a recent and emerging application domain of WSNs. Consequently, the application of WSNs in smart grids arise new constraints that should be managed by WSN protocols. As summarized in Fig. 1, these constrains pertain to (i.) the harsh smart grid environments, (ii.) the different QoS requirements of WSN-based smart grid applications (mainly in 


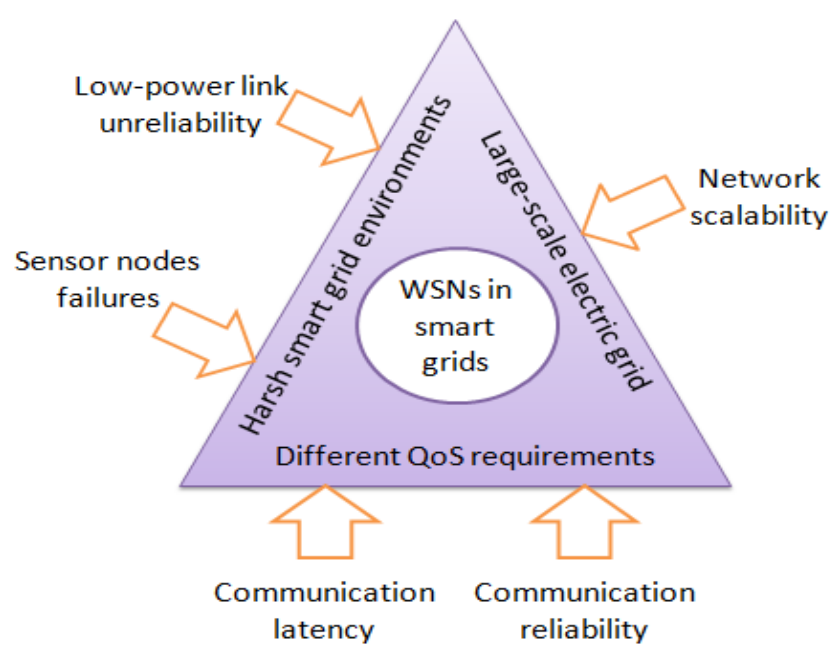

Fig. 1 Constraints of WSN-based smart grid applications.

terms of communication reliability and latency), and (iii.) the large-scale of the electric grid. In this section, we discuss these constraints, while emphasizing their impact on the design of efficient WSN communication protocols for smart grid applications.

\subsection{Harsh smart grid environments}

Electric grid environments are typically characterized by highly corrosive conditions (e.g. wind, rain, solar radiation, humidity, vibrations, dust, etc.), which contributes to communication link unreliability and sensor nodes failures.

Low-power link unreliability in smart grid environments. Communication links in WSNs (also referred as low-power links) have been shown to be extremely unreliable: Their quality fluctuates over time and space, and connectivity is typically asymmetric $[8,9]$. This is due to the low cost and low-power radios used in WSNs. Unfortunately, the harsh smart grid environments make low-power links even more unreliable. For example, Gungor et al. [30] argue that smart grid distribution environments have variable link delivery and higher packet error rate due to electromagnetic interference, obstructions, equipment's noise, etc. Low-power link unreliability in smart grid environments constitutes a major handicap for communication protocols to maintain the network correct operation and ensure acceptable end-to-end data delivery.

Link quality estimation enables network protocols to overcome low-power link unreliability. For instance, some routing protocols rely on link quality estimation to deliver data over links with high quality, which improves the network throughput by limiting packet loss and maximizes its lifetime by minimizing the number of retransmissions and avoiding route re-selection triggered by links' failure. Topology control mechanisms also rely on link quality estimation to maintain the stability of the topology. High-quality links are long-lived, therefore, efficient topology control 
mechanisms aggregate high-quality links to maintain robust network connectivity for long periods, thus avoiding unwanted transient topology breakdown.

Empirical characterization of low-power links through real-world measurements in smart grid environments is also of paramount importance to mitigate low-power link unreliability. It allows a deep and clear understanding of link behavior and statistical properties, which (1) contributes to the design of efficient link quality estimators, and (2) allows deriving accurate wireless channel models that reflect radio propagation in real smart grid environments. For example, in [30], based on extensive empirical link measurements the authors provided an estimation of the parameters of the log-normal shadowing path loss model, for different power distribution environments.

Sensor failures in harsh smart grid environments. The severe conditions of electrical grids may adversely affect the performance of sensor nodes, causing a portion of them to malfunction. Further, sensor devices can be deployed in locations that are tough to access and replace battery (e.g. sensors for underground cables) [16].

Sensor failures and battery depletion impact the network topology and can be overcome using adaptive protocols to maintain the network operation following node failures. Energy harvesting techniques may be also used to extend the battery lifetime of sensor nodes. In some WSN-based smart grid applications, main power may be available. For instance, in [26], the power supply to the sensor nodes is obtained from power supplies in substations and power lines. However, the voltages of the power lines are too high and should be stepped down before being used by the sensor nodes.

\subsection{Different Quality of Service (QoS) requirements}

WSNs are expected to satisfy the main requirements of smart grid communications, namely reliability and latency. On the other hand, WSN-based smart grid applications have different and often contradictory QoS requirements in terms of reliability and latency, resulting in a heterogeneous data traffic patterns [32]. For example, overhead transmission line monitoring and substation automation represent critical smart grid applications that need strict reliability and latency requirements, while other applications, such as Demand Response and AMI, do not expect critical latency. Some other smart grid applications, such as residential energy management, can occasionally tolerate losses in data transfer.

The heterogeneity of traffic makes QoS provisioning in WSN-based smart grid applications a non-trivial task. Data traffic should be classified into several classes, according to the QoS requirements. Communication protocols are then expected to provide differentiated services to handle different traffic classes originated from a wide range of possible smart grid applications [5]. Service differentiation is discussed in Section 5.1.

\subsection{Large-scale electric grid}

The geographical span of the electric grid is anticipated to be very large, especially with its extension into the consumer premises [16]. The number of sensors 
employed to monitor the different smart grid components, from generation to consumer side, is too high and the management of this large-scale network would be a challenging task [65]. For this reason, the design of scalable communication protocols for WSN-based smart grid applications is necessary (e.g., IP-based protocols). Data aggregation techniques are also mandatory to avoid redundant data collected by sensors close to each other.

\section{On the optimization of communication protocols for WSN-based smart grid applications}

The application of WSNs in smart grids brought new challenges that should be properly considered by communication protocols. A fundamental question is

"whether existing WSN communication protocols and standards are adequate for smart grid applications?". Several research efforts have been dedicated for providing response elements to this question. For example, in [11], Bilgin et al. investigated the performance of ZigBee in different smart grid environments. It was shown that ZigBee is only adequate for low-data rate smart grid applications not requiring high reliability and real time deadlines. Temel et al. [61] studied the performance of different types of routing protocols, namely on-demand (AODV (Ad hoc On-Demand Distance Vector) and DYMO (Dynamic MANET On-demand)), table-driven (DSDV (Destination Sequenced Distance Vector)) and QoS-aware (TUQR (Topology Unaware QoS Routing)) protocols. They demonstrated that these routing protocols are unable to deliver satisfactory performance in smart grid environments [61]. The performance of state-of-the art MAC protocols, namely IEEE 802.15.4, IEEE 802.11, CSMA, TDMA and Z-MAC, was evaluated by Yigit et al. [68], demonstrating that these MAC protocols can not deliver adequate performance in smart grid environments when network traffic load increases [68]. As a conclusion, traditional WSN protocols have been shown not sufficiently adequate for smart grid applications having different QoS requirements.

Recently, several techniques and mechanisms have been introduced for the optimization of existing WSN communication protocols for smart grid applications, especially (1) traffic/service differentiation, (2) interference mitigation and (3) link quality estimation. In this Section, we classify the recent research works on the optimization of WSN protocols for smart grid applications according to the above mentioned optimization techniques as well as the targeted layer (i.e., layered and cross-layer approaches). Table 1 illustrates the proposed taxonomy.

\subsection{Traffic/service differentiation}

Service differentiation is a mechanism allowing distinguishing different traffic classes where each class has its specific QoS requirements and priorities (in terms of reliability, latency, throughput, etc.). As stated previously, WSN-based smart grid applications are diverse which leads to various traffic patterns, each having its own QoS requirements (e.g., smart meter readings, real time pricing information, periodic monitoring of electric components, etc.). Therefore, QoS differentiation in WSN-based smart grid applications is a fundamental building block for com- 
munication protocols to ensure acceptable QoS with respect to each application or traffic class.

QoS differentiation can be performed at different layers, including network [ 54 , $50]$ and MAC $[2,3,60,4]$ layers. It can also be performed at several layers. It is thus referred to as a cross-layer QoS optimization scheme, such as in [60]. The proposed solution consists of a MAC and a routing algorithm with QoS support. At the MAC layer, the existing CSMA/CA of IEEE 802.15.4 was improved by providing differentiated services for data traffic with different priorities (low and high). The introduced routing algorithm cooperates with physical and MAC layers to select paths that satisfy the reliability and latency requirements.

\subsubsection{Routing level QoS differentiation}

The authors in [54] argued that multi-path routing, i.e., routing that establishes multiple paths between the source and the destination, provides QoS differentiation in WSN-based smart grid applications. To demonstrate their statement, the authors performed a comparative performance study of two WSN routing protocols: SPEED and MMSPEED. SPEED is a single-path routing protocol that does not support QoS differentiation. However, it is a QoS-aware protocol as it provides end-to-end communication deadline guarantees. MMSPEED extends SPEED by providing QoS differentiation through multi-path routing. In addition to real-time communication guarantees, MMSPEED guarantees different reliability requirements by enabling probabilistic multi-path forwarding.

Different from the previous work (which provides QoS differentiation through multi-path routing), a QoS differentiation mechanism that exploits multi-topology routing in RPL protocol, is presented in [50]. In RPL, a network topology consists of one or several DODAGs (Destination-Oriented Directed Acyclic Graph), which form together an RPL instance. Each instance is built based on an Objective Function, which specifies how to select paths based on certain criteria (e.g., link quality, latency, hop count, etc.). An RPL network may consist of multiple RPL instances where each instance operates independently of the others. This feature was exploited in [50] to provide QoS differentiation for RPL, deployed in smart grid Neighbor Area Networks (NANs). The authors proposed to associate each traffic type with a different RPL instance. Especially, Objective Functions based on Expected Transmission Count (ETX) and Hop Count (HC) were associated with critical traffic and periodic traffic, respectively.

\subsubsection{MAC level QoS differentiation}

In [2-4], QoS differentiation is performed at MAC level by optimizing the IEEE 802.15.4. In [2], the authors targeted high data rate smart grid monitoring applications, such as Partial Discharge (PD) monitoring. The adoption of the IEEE 802.15.4 standard for these applications can lead to excessive delays as it is designed for low data rate applications. The authors in [2] introduced a QoS differentiation scheme that reduces the end-to-end delay of high priority and delay critical packets. The introduced solution is based on a delay optimization model of IEEE 802.15.4 for cluster-tree WSNs. The model allows each Cluster Head (CH) minimizing the inter- $\mathrm{CH}$ delay by adjusting two IEEE 802.15.4 MAC parameters: the Contention Access Period (CAP) and the CFP (Contention Free Period). 
Another optimization of the IEEE 802.15.4 MAC is presented in [4], where the authors introduced two adaptive delay reduction schemes for cluster-tree and mesh WSNs. The proposed schemes can adaptively tune the Guaranteed Time Slot (GTS) to provide QoS differentiation required by delay critical smart grid applications.

To address latency and QoS differentiation requirements in smart grid monitoring applications, the authors in [3] proposed a scheme that is based on data prioritization and delay estimation procedures performed at the application layer. Thus, service differentiation is provided to high priority traffic by modifying the Clear Channel Assessment (CCA) duration in the IEEE 802.15.4.

\subsection{Interference mitigation}

Smart grid environments are commonly characterized by electromagnetic interference, equipment's noise and multi-path effects, which negatively impact the performance of WSN communications [30]. Two techniques have been proposed to mitigate the impact of interference in smart grid environments: cognitive radio technology and multi-channel communication.

\subsubsection{Cognitive radio technology}

It is relatively a new approach for utilizing and accessing spectrum (i.e., radio frequencies) in smart ways. Cognitive radios have the ability to dynamically sense the spectrum, determine the unused bands and exploit them in an opportunistic manner. Consequently, they can identify potential troubles in communication quality, such as interference and multi-path fading. These features make cognitive radio technology a promising solution for WSN applications in smart grid environments to overcome their hostile propagation conditions [28]. Cognitive radio sensor networks can enhance the network performance in terms of reliability and communication latency through the mitigation of the congested and noisy bands in the spectrum. This allows the selection of high capacity links for wireless communications in smart grids and hence improves the reliability and the latency of the network. For example, the authors in [56,55], exploited cognitive radio communications to circumvent the harsh propagation conditions in the smart grid. They proposed a cognitive radio-based framework that supports the QoS requirements of WSN-based smart grid applications, namely latency, data rate and reliability.

However, the benefits of cognitive radio come with significant additional energy consumption mainly caused by spectrum sensing operations, which may affects the network lifetime. The advantages and challenges of cognitive radio wireless sensor networks in smart grid applications are discussed in detail in [10].

\subsubsection{Multi-channel communication}

Multi-channel mechanism allows several nodes to communicate at the same time, using different channels $[36,59]$. Hence, multi-channel WSNs allow to (i.) alleviate the effects of interference on wireless medium and (ii.) achieve parallel transmissions through multiple channels for better data delivery. Multi-channel communication has been argued to be an efficient solution for WSN-based smart grid appli- 
cations to mitigate electromagnetic interference, equipment's noise and multi-path effects characterizing smart grid environments.

In [12], the performance of multi-channel MAC protocols was evaluated in different smart grid environments and compared against that of single channel WSN MAC protocols. This study showed that multi-channel WSNs improve the network capacity compared to single channel WSNs: Increasing the number of communication channels helps to increase the packet reception rate, reduce communication delay and increase almost twice the network throughput. 
Table 1 Taxonomy of contributions related to the optimization of WSN communication protocols for smart grid applications

\begin{tabular}{|c|c|c|c|c|c|c|}
\hline \multirow[t]{2}{*}{ Works } & \multicolumn{2}{|c|}{ Layered approach } & \multirow{2}{*}{$\begin{array}{l}\text { Cross-layer } \\
\text { approach }\end{array}$} & \multicolumn{3}{|c|}{ Optimization technique } \\
\hline & MAC layer & Routing layer & & $\begin{array}{l}\text { Traffic/service } \\
\text { differentiation }\end{array}$ & $\begin{array}{l}\text { Interference mit- } \\
\text { igation }\end{array}$ & $\begin{array}{l}\text { Link quality esti- } \\
\text { mation }\end{array}$ \\
\hline$[2]$ & $\begin{array}{l}\text { Optimization } \\
\text { of the IEEE } \\
802.15 .4 \text { MAC } \\
\text { for high data } \\
\text { rate applications }\end{array}$ & & & $\checkmark$ & $\mathrm{x}$ & $\mathrm{x}$ \\
\hline [54] & & $\begin{array}{lr}\begin{array}{l}\text { Use of } \\
\text { path }\end{array} & \text { routti- } \\
\text { protocoling } & \\
\end{array}$ & & $\checkmark$ & $\mathrm{x}$ & $\begin{array}{l} \\
\text { LQE: PRR }\end{array}$ \\
\hline$[50]$ & & $\begin{array}{l}\text { Use of multiple } \\
\text { RPL instances }\end{array}$ & & $\checkmark$ & $\mathrm{x}$ & $\begin{array}{l}\checkmark \\
\text { LQE: ETX }\end{array}$ \\
\hline [29] & & $\begin{array}{l}\text { Impact of repre- } \\
\text { sentative LQEs } \\
\text { on CTP routing } \\
\text { protocol }\end{array}$ & & $\begin{array}{ll} & \\
\text { (QoS provi- } \\
\text { sioning for } 1 \\
\text { traffic class) }\end{array}$ & $\mathrm{x}$ & $\begin{array}{l}\checkmark \\
\text { LQEs: PRR, } \\
\text { WMEWMA, } \\
\text { RNP, ETX and } \\
\text { four-bit }\end{array}$ \\
\hline$[52]$ & & $\begin{array}{l}\text { Use of holistic } \\
\text { link quality es- } \\
\text { timation to im- } \\
\text { prove the reli- } \\
\text { ability of RPL } \\
\text { routing protocol }\end{array}$ & & $\begin{array}{l}\text { x } \\
\text { (QoS provi- } \\
\text { sioning for } 1 \\
\text { traffic class) }\end{array}$ & $\mathrm{x}$ & $\begin{array}{l}\checkmark \\
\text { LQE: } \\
\text { FLQE }\end{array}$ \\
\hline$[67]$ & & & $\begin{array}{l}\text { Use of multi- } \\
\text { channel MAC \& } \\
\text { Tree routing }\end{array}$ & $\begin{array}{ll}x & \\
\text { (QoS provi- } \\
\text { sioning for } 1 \\
\text { traffic class) } \\
\end{array}$ & $\begin{array}{l}\checkmark \\
\text { Technique: } \\
\text { multi-channel } \\
\text { communication }\end{array}$ & $\begin{array}{l}\checkmark \\
\text { LQE: PRR }\end{array}$ \\
\hline$[66]$ & & & $\begin{array}{l}\text { Use of multi- } \\
\text { channel MAC \& } \\
\text { channel-aware } \\
\text { routing }\end{array}$ & $\checkmark$ & $\begin{array}{l}\checkmark \\
\text { Technique: } \\
\text { multi-channel } \\
\text { communication }\end{array}$ & $\begin{array}{l}\checkmark \\
\text { LQE: PRR }\end{array}$ \\
\hline$[56][55]$ & & & $\begin{array}{l}\text { Design of a } \\
\text { framework for } \\
\text { QoS support } \\
\text { (interaction of } \\
\text { routing, MAC } \\
\text { and physical } \\
\text { layers) }\end{array}$ & $\checkmark$ & $\begin{array}{l}\checkmark \\
\text { Technique: } \\
\text { cognitive radio } \\
\text { technology }\end{array}$ & $\mathrm{x}$ \\
\hline$[60]$ & & & $\begin{array}{lr}\text { An } & \text { improved } \\
\text { IEEE } 802.15 .4 \\
\text { MAC \& Routing } \\
\text { algorithm with } \\
\text { QoS differentia- } \\
\text { tion }\end{array}$ & $\checkmark$ & $\mathrm{x}$ & $\begin{array}{l}\checkmark \\
\text { LQE: PRR }\end{array}$ \\
\hline [3] [4] & & & $\begin{array}{l}\text { Adaptation of } \\
\text { IEEE } 802.15 .4 \\
\text { MAC (interac- } \\
\text { tion of MAC } \\
\text { and application } \\
\text { layers) }\end{array}$ & $\checkmark$ & $\mathrm{x}$ & $\mathrm{x}$ \\
\hline
\end{tabular}


Although multi-channel communications allows mitigating interference, recent research studies show that the network capacity is still limited by the routing topology $[67,66]$. In [67], the authors proposed the use of both multi-channel communication together with an appropriate routing tree protocol to further enhance the performance of WSNs in smart grid environments. They selected RBCA (ReceiverBased Channel Assignment) as a multi-channel MAC algorithm. The network performance was evaluated using different routing tree algorithms, demonstrating that CMST (Capacitated Minimum Spanning Tree) with PRR routing tree shows an improvement in the network capacity in terms of delay and throughput.

In [66], the authors modified the RBCA's time slot assignment algorithm as well as the transmission schedule in order to support data prioritization. They proposed a priority and channel-aware scheduling algorithm for smart grid applications. The proposed algorithm performs successively (i.) channel assignment, while considering interference between nodes, (ii.) time slot assignment and (iii.) delay-aware data transmission, allowing packet transmission according to their traffic classes and priorities. Three classes of traffic were considered: real-time, non-real-time and best effort traffic, having high, medium and low priority respectively. The real-time packets are transmitted first, then the non-real-time and best effort packets are sent respectively.

\subsection{Link quality estimation}

Link quality estimation is a prerequisite for higher layer protocols to overcome low-power links unreliability in smart grid environments [30]. The performance of well-known Link Quality Estimators (LQEs) in typical smart grid environments was studied in [29]. The evaluated LQEs are Packet Reception Ratio (PRR), Window Mean with Exponentially Weighted Moving Average (WMEWMA), Expected Transmission Count (ETX), Required Number of Packet Transmissions (RNP) and four-bit. The comparative simulation study is based on radio propagation parameters of typical smart grid environments, found experimentally in [30]. The performance of LQEs is assessed by comparing their impact on the collection tree routing protocol (CTP). The authors found that ETX and four-bit estimators showed the best performance in different smart grid environments since they consider the link asymmetry property.

The authors in [51] compared the performance of LQEs, using a different evaluation methodology from that used in [29]. This methodology consists in analyzing the statistical properties of LQEs, independently from any external factors like MAC collisions or routing. Further, the authors in [51] devised using composite LQEs (i.e., LQEs that combine several link metrics) to provide holistic and reliable link quality estimation for WSN-based smart grid applications. The evaluated LQEs are ETX, four-bit and F-LQE (Fuzzy-Link Quality Estimator). It was found that, F-LQE is more reliable and more stable than ETX and four-bit. However, it lacks reactivity which makes it not sufficiently adequate for smart grid environments characterized by excessive packet loss and link burstiness. An optimized version of F-LQE, called Opt-FLQE, that improves the reactivity and reduces the computational complexity of the original F-LQE is then proposed.

In [52], the authors used Opt-FLQE for reliable end-to-end communication in RPL networks deployed in smart grids. They proposed an alternative routing 
metric for RPL based on Opt-FLQE, called Opt-FLQE ${ }_{R M}$. The authors showed that Opt-FLQE $E_{R M}$ improves RPL performance over traditional routing metrics, including the RPL default metric, mainly in terms of packet loss ratio and endto-end delay.

\subsection{Discussion and open issues}

The application of WSNs in smart grids raises new constraints, pertaining to both the harsh electrical grid nature and the diverse QoS requirements of smart grid monitoring applications. Throughout this section, we have presented relevant techniques that have been introduced to cope with these constraints, namely QoS differentiation, interference mitigation (through multi-channel communication or cognitive radio communication) and link quality estimation. Nevertheless, the deployment of WSNs in smart grids still has several challenges and open issues as detailed next:

- Energy efficiency of cognitive radio sensor networks in smart grid communications. Cognitive radio technology promises minimizing the inconvenient environmental effects of smart grids (e.g. interference) and then improving the communication delay and reliability of WSNs in smart grids. However, the proposed approaches based on cognitive radio technology $[10,56,55]$ have not been evaluated in terms of energy-efficiency. It is well known that, the spectrum scanning operation performed by the cognitive radio is energy greedy [28] and thus, achieving energy-efficient communication, especially in large scale smart grid deployments, is not a trivial task. Therefore, there is a need for further research efforts addressing the trade-off between energy consumption, reliability and communication delay. On the other hand, the scarcity of WSN platforms with cognitive radio capabilities justifies the fact that most related literature is simulation-based. Common WSN radio chips (e.g. the CC1000 radio used for Mica2 nodes and the CC2420 radio for MICAz and TelosB sensor nodes) are not cognitive radios.

- Adoption of standard multi-channel MAC protocols for WSN-based smart grid. Multi-channel communication technique is supported by the available radios, such as the CC2420 radio used in MICAz and TelosB WSN platforms. These radios can just be tuned to operate on multiple frequency channels. However, the performance of WSN multi-channel protocols is not well investigated, especially for standardized multi-channel MAC protocols that offer the ease of integration and interoperability, as well as communication reliability.

The IEEE 802.15.4e standard [35] is a recent amendment of IEEE 802.15.4 MAC specification, that aims at meeting the requirements (reliability, ultra-low power consumption and bounded latency) of industrial applications, including smart grid applications. It enhances the old standard by introducing mechanisms such as multi-channel communication, time slotted access and channel hopping. The IEEE 802.15.4e defines several MAC operation modes, including Time Slotted Channel Hopping (TSCH) and Deterministic and Synchronous Multi-channel Extension (DSME) [49,27]. Both TSCH and DSME MAC protocols enhance communication reliability by employing channel diversity schemes and time slotted access. To improve reliability, TSCH MAC protocol employs 
channel hopping, while DSME MAC protocol employs two types of channel diversity schemes: channel hopping and channel adaptation. The suitability and the adoption of TSCH and DSME MAC protocols for WSN-based smart grid applications is still an open research issue.

- Accurate link quality estimators for WSN higher layer protocols in smart grid applications. Link quality estimation is a prerequisite for higher layer protocols to improve the end-to-end data delivery and overcome low-power link unreliability in harsh smart grid environments. We notice that most approaches proposed for WSN-based smart grid applications leverage on simple link quality estimators, such as PRR, [54,60,67,66]. However, it is now well known that PRR is inaccurate as it is only able to provide one link aspect, link delivery, and ignores other important aspects such as link asymmetry [8]. Thus, there is a need for employing accurate link quality estimators which are able to reflect the real link status in smart grid environments. Further, few of work (except [30]) tackled the empirical analysis of low-power links in smart grid environments. Such studies are of paramount importance as they provide a better understanding of low-power link characteristics, which contributes to the design of accurate link quality estimators and realistic wireless channel models improving network simulation as it will be discussed in the next Section.

- Real experimentation of WSNs in smart grid environments. Unfortunately, most research studies around WSN-based smart grid applications are based on network simulation, using channel parameters of typical smart grid distribution environments derived in [30]. Definitely, the study in [30] represents a valuable contribution to the state of the art. However, it would be interesting to conduct additional similar studies to derive channel parameters in other smart grid environments which are more hostile, especially in the smart grid generation side such as power plants. Generally, there is a clear gap in literature in what concerns real experimentation of WSNs in smart grids.

\section{Validation platforms for WSN-based smart grid solutions}

Experimental evaluation through field trials is an efficient validation tool, as it allows anticipating the deployment of the proposed WSN solutions in real smart grid environments and then get performance results with high confidence. However, conducting field trials in harsh smart grid environments is not a trivial task. For instance, performing experiments at the generation and transmission sides of the smart grid (e.g., power plants and substations), where the voltages are too high, can endanger the human safety. Running real experiments in laboratory smart grid Testbeds can be an interesting alternative. However, up to now, publicly accessible smart grid Testbeds are still missing.

Due to the challenges raised by field trials, most research efforts on WSNbased smart grid solutions use network simulation. However, to reflect smart grid features and get meaningful results, simulation scenarios should be properly set using channel parameters that reflect radio propagation behavior in smart grid environments. Generally, these parameters pertain to a particular radio propagation model implemented in the simulator. For instance, the log normal shadowing path loss model has been argued as an accurate radio propagation model $[29,67]$ and has been widely adopted in WSN-based smart grid $[11,61,54,51]$. The parameters 
of this model have been estimated in typical smart grid distribution environments in [30], namely an outdoor $500 \mathrm{kV}$ substation, an indoor main power control room and an underground transformer vaults. Simulators such as NS-2 and TOSSIM rely on this radio propagation model. Hence, performing simulation with these simulators while setting the channel parameters to realistic values such as those provided by Gungor et al. [30] contributes to rigorous simulation results.

The choice of the convenient network simulator to validate WSN-based smart grid communication protocols depends not only on the available radio propagation models, but also on the implemented MAC and routing protocols. In order to help network designers to select the adequate WSN simulator, an overview and a comparison of the potential simulators for WSN-based smart grid applications is given next.

\subsection{Potential simulators for WSN-based smart grid applications}

In this section, we present the most representative WSN simulators that have been used to validate WSN-based smart grid solutions. Table 2 presents some technical features of these simulators.

- NS-2 (Network Simulator 2) [14] is a general network simulator (non-specific for WSNs). It is implemented in $\mathrm{C}++$ and the simulation scripts are written in OTcl (an object-oriented extension of Tcl (Tool Command Language)). A considerable number of communication protocols are already implemented in NS-2, which make it a very popular simulator.

NS-2 is the most used simulator in the community of WSN-based smart grid applications $[11,61,68]$. One of the reasons is the accuracy of its radio model namely the log normal shadowing path loss model.

- NS-3 [33] is a subsequent version of NS-2. It brings several improvements compared to NS-2, regarding modularity, re-usability and extensibility. NS-3 is entirely developed in $\mathrm{C}++$, including simulation scripts, which can be also developed in Python. It is important to notice that NS-2 simulation scripts can not be run in NS-3 as they are written in OTcl.

- TOSSIM [39] is a WSN simulator available as part of TinyOS operating system, written in nesC (network embedded system C) programming language. The same TinyOS applications, which are written in nesC, can be run on real WSN nodes as well as simulated nodes (MICAz motes). The simulation scenario can be defined in Python or $\mathrm{C}++$. A configurable CSMA-like MAC protocol is used by TOSSIM where several properties, such as backoffs and delays, can be adjusted. To simulate the signal propagation between nodes, TOSSIM uses the log-normal shadowing path loss model.

- COOJA [48] is a Java-based WSN simulator/emulator available as part of Contiki operating system [15]. COOJA is included in Instant Contiki ${ }^{1}$, an entire development environment that can be easily downloaded as a virtual machine. Like TOSSIM, the same Contiki application code, written in C, can run on both real and simulated sensor nodes. Contiki provides a compact implementation of the IPv6 communication stack optimized for embedded devices, called $\mu$ IPv6.

\footnotetext{
1 http://www.contiki-os.org/start.html
} 
This protocol stack includes the 6LoWPAN standard and the RPL routing protocol. One limitation of COOJA is that it supports simplistic radio models such as the unit disk graph model.

- J-Sim $[57,58]$ is a network simulator entirely implemented in Java. J-Sim latest release (version 1.3) defines simulation script in Jacl (a Java implementation of Tcl script language). It has been extended to include frameworks for WSNs simulation, including an implementation of AODV and DSR routing protocols. J-Sim integrates simplistic radio propagation models, namely Free-space, Tworay ground and Irregular terrain models. The authors in [54] implemented the log-normal shadowing path loss model in order to accurately model the wireless channel in typical smart grid environments.

- QualNet [37] is a commercial network simulator based on $\mathrm{C}++$. It is designed for large-scale networks (i.e., thousands of nodes can be modeled). This feature is provided by the use of Parsec programming language (an extension of $\mathrm{C}$ language for parallel programming).

- OMNeT++ (Objective Modular Network Testbed in C++) [62] is a C++-based simulator destined to model communication networks and distributed and/or parallel systems. The network and topology descriptions in OMNeT ++ are developed in Network Description (NED) language.

Castalia [13] is a simulation framework that extends OMNeT++ for WSNs. Castalia provides a configurable MAC protocol (where some parameters can be adjusted) and an implementation of T-MAC and S-MAC protocols. However, it supports only simple routing protocols, namely Multipath Rings and simple Tree.

\subsection{Discussion and open issues}

The design of network protocols tailored to the specific characteristics of the WSNbased smart grid monitoring applications (presented in Section 4) is relatively a new research area and the validation of such protocols is still a challenging task.

Simulation is a widely considered validation technique in the literature. Sophisticated network simulators for WSN-based smart grid applications should basically integrate (1) representative WSN protocols and standards, such as 6LoWPAN, Zigbee and IEEE 802.15.4, and also (2) accurate radio propagation models such as the log normal shadowing path loss model [30,67]. Further, simulation scenarios should be properly set using channel parameters reflecting real WSN applications is smart grid environments. Unfortunately, there is a lack of such sophisticated network simulators. Therefore, an important and urgent open issue is to optimize existing simulators to get convenient validation tools for WSN-based smart grid applications. For example, COOJA is a well known simulator that has been extensively used in WSN research community thanks to its IPv6 protocol stack. However, its radio model is very simplistic. Hence, there is a need to implement the log normal shadowing path loss model for COOJA simulator.

The use of the co-simulation technique represents another potential validation tool for WSN protocols in smart grid applications. Co-simulation generally refers to the cooperation of two or more simulators. In the case of smart grids, power/network co-simulation mandates the integration of a power simulator and a network simulator. Co-simulation is expected to allow studying and validating 
WSN-based smart grid solutions in a near real electric grid behavior. Different smart grid co-simulation platforms were proposed, including [40,70,41]. For instance, GECO (Global Event-driven CO-simulation platform) [41] is an example of co-simulation platform where the power system is modeled using PSLF and integrated with NS-2 network simulator. However, the different proposed co-simulators are not yet widely used by researchers. This may be due to the fact that they are just prototype tools (some implementations are not available to the research community as an open-source). Further, the co-simulation configuration may be a complex process that requires a deep knowledge about electrical components modeling. Therefore, the usefulness of co-simulation as a validation tool for WSN protocols in smart grids needs to be demonstrated. 
Table 2 Comparison of potential simulators for WSN-based smart grid applications

\begin{tabular}{|c|c|c|c|c|c|}
\hline \multicolumn{2}{|c|}{ Simulator } & $\begin{array}{l}\text { Programming/ } \\
\text { Simulation } \\
\text { language }\end{array}$ & $\begin{array}{l}\text { Supported MAC } \\
\text { protocols }\end{array}$ & $\begin{array}{l}\text { Supported rout- } \\
\text { ing protocols }\end{array}$ & $\begin{array}{lll}\text { Supported } & \text { radio } & \text { propagation } \\
\text { models } & & \end{array}$ \\
\hline \multirow[t]{2}{*}{ NS } & NS-2 & $\mathrm{C}++/ \mathrm{OTcl}$ & $\begin{array}{lr}\text { IEEE } & 802.11 \\
\text { MAC, } & \text { TDMA, } \\
\text { IEEE } & 802.15 .4 \\
\text { MAC } & \end{array}$ & $\begin{array}{l}\text { AODV, DSDV, } \\
\text { DSR, TORA }\end{array}$ & $\begin{array}{l}\text { Free space, Two-ray ground reflection, } \\
\text { Log normal shadowing }\end{array}$ \\
\hline & NS-3 & C++ / Phyton & $\begin{array}{l}\text { IEEE } 802.11 \text { MAC, } \\
\text { CSMA }\end{array}$ & $\begin{array}{l}\text { AODV, DSDV, } \\
\text { DSR, OLSR }\end{array}$ & $\begin{array}{l}\text { Fixed Received Signal Strength Propa- } \\
\text { gation, Matrix Propagation Loss, Ran- } \\
\text { dom Propagation Loss, Okumura Hata } \\
\text { Propagation, Friis Propagation, Log } \\
\text { Distance Propagation, Three Log Dis- } \\
\text { tance Propagation, Two Ray Ground } \\
\text { Propagation (ported from NS-2), Jakes } \\
\text { propagation, Nakagami propagation. }\end{array}$ \\
\hline \multicolumn{2}{|c|}{ TOSSIM } & $\begin{array}{l}\text { NesC } \\
\text { Python, } \mathrm{C}++\end{array}$ & $\begin{array}{l}\text { Configurable } \\
\text { CSMA-like MAC }\end{array}$ & CTP, DYMO & Log normal shadowing \\
\hline \multicolumn{2}{|c|}{ COOJA } & Java / C & $\begin{array}{l}\text { IEEE } \\
\text { 802.15.4 MAC } \\
(\text { CSMA/CA), } \\
\text { NullMAC } \\
\end{array}$ & RPL, AODV & $\begin{array}{l}\text { Unit Disk Graph, Directed graph, } \\
\text { Multi-path ray tracing }\end{array}$ \\
\hline \multicolumn{2}{|c|}{ J-Sim } & Java / Tcl & IEEE $802.11 \mathrm{MAC}$ & AODV, DSR & $\begin{array}{l}\text { Free space model, Two-ray ground } \\
\text { model, Irregular terrain model }\end{array}$ \\
\hline \multicolumn{2}{|c|}{ QualNet } & Parsec $\mathrm{C}++$ & $\begin{array}{l}\text { Point-to-point, } \\
\text { CSMA, IEEE } \\
802.11 \text {, IEEE } 802.3\end{array}$ & $\begin{array}{l}\text { AODV, DSR, } \\
\text { LAR1, ODMRP }\end{array}$ & $\begin{array}{l}\text { Free space model, two ray path } \\
\text { loss model, Irregular Terrain Model, } \\
\text { Ricean model, Rayleigh model, Con- } \\
\text { stant shadowing model, Log normal } \\
\text { shadowing model }\end{array}$ \\
\hline \multicolumn{2}{|c|}{ OMNeT $++/$ Castalia } & $\mathrm{C}++/ \mathrm{NED}$ & \begin{tabular}{l}
\multicolumn{2}{l}{ Configurable } \\
MAC, T-MAC, \\
S-MAC
\end{tabular} & $\begin{array}{l}\text { Simple Tree, Multi- } \\
\text { path Rings }\end{array}$ & $\begin{array}{l}\text { Free space, Log normal shadowing, } \\
\text { Temporal variation }\end{array}$ \\
\hline
\end{tabular}




\section{Conclusion}

The equipment failures and the limited monitoring and control capabilities in the current electric grid are the main motivations for the migration to a smarter grid with advanced communication and monitoring skills. WSNs represent a promising technology for enabling the pervasive monitoring and control in smart grids. However, the application of WSN communication technology in smart grids brought new challenges, that should be managed by WSN protocols.

In this paper, we identified and discussed these arisen challenges. Then, we proposed a thorough taxonomy of recent research works on the optimization of existing WSN communication protocols, to cope with the new constraints emerged from the deployment of WSNs in smart grid applications. We also highlighted open issues and discussed the potential validation platforms for WSN-based smart grid applications.

\section{References}

1. Al-Anbagi, I.S., Erol-Kantarci, M., Mouftah, H.T.: A delay mitigation scheme for WSNbased smart grid substation monitoring. In: IWCMC, pp. 1470-1475 (2013)

2. Al-Anbagi, I.S., Erol-Kantarci, M., Mouftah, H.T.: QoS-aware inter-cluster head scheduling in WSNs for high data rate smart grid applications. In: 2013 IEEE Global Communications Conference, GLOBECOM 2013, Atlanta, GA, USA, December 9-13, 2013, pp. 2628-2634 (2013)

3. Al-Anbagi, I.S., Erol-Kantarci, M., Mouftah, H.T.: Priority- and delay-aware medium access for wireless sensor networks in the smart grid. IEEE Systems Journal 8(2), 608618 (2014). DOI 10.1109/JSYST.2013.2260939

4. Al-Anbagi, I.S., Erol-Kantarci, M., Mouftah, H.T.: Delay critical smart grid applications and adaptive qos provisioning. IEEE Access 3, 1367-1378 (2015). DOI 10.1109/ACCESS.2015.2466077

5. Al-Anbagi, I.S., Erol-Kantarci, M., Mouftah, H.T.: A survey on cross-layer quality-ofservice approaches in WSNs for delay and reliability-aware applications. IEEE Communications Surveys and Tutorials 18(1), 525-552 (2016). DOI 10.1109/COMST.2014.2363950

6. Al-Anbagi, I.S., Mouftah, H.T., Erol-Kantarci, M.: Design of a delay-sensitive WSN for generation monitoring in the smart grid. In: CCECE, pp. 1370-1373 (2011)

7. Ancillotti, E., Bruno, R., Conti, M.: The role of communication systems in smart grids: Architectures, technical solutions and research challenges. Computer Communications 36(17-18), 1665-1697 (2013)

8. Baccour, N., Koubaa, A., Mottola, L., Zamalloa, M.A.Z., Youssef, H., Boano, C.A., Alves, M.: Radio link quality estimation in wireless sensor networks: A survey. TOSN 8(4), 34 (2012)

9. Baccour, N., Koubaa, A., Noda, C., Fotouhi, H., Alves, M., Youssef, H., Zuniga, M., Boano, C.A., Römer, K., Puccinelli, D., Voigt, T., Mottola, L.: Radio Link Quality Estimation in Low-Power Wireless Networks. Springer Briefs in Electrical and Computer Engineering. Springer (2013). DOI 10.1007/978-3-319-00774-8

10. Bicen, A., Akan, O., Gungor, V.: Spectrum-aware and cognitive sensor networks for smart grid applications. Communications Magazine, IEEE 50(5), 158-165 (2012). DOI 10.1109/MCOM.2012.6194397

11. Bilgin, B.E., Çagri Güngör, V.: Performance evaluations of zigbee in different smart grid environments. Computer Networks 56(8), 2196-2205 (2012). DOI 10.1016/j.comnet.2012.03.002

12. Bilgin, B.E., Gungor, V.C.: On the performance of multi-channel wireless sensor networks in smart grid environments. In: ICCCN, pp. 1-6 (2011)

13. Boulis, A.: Castalia: revealing pitfalls in designing distributed algorithms in wsn. In: Proceedings of the 5th international conference on Embedded networked sensor systems, pp. 407-408. ACM (2007)

14. Downard, I.T.: Simulating sensor networks in NS-2. Tech. rep., DTIC Document (2004) 
15. Dunkels, A., Gronvall, B., Voigt, T.: Contiki-a lightweight and flexible operating system for tiny networked sensors. In: Local Computer Networks, 2004. 29th Annual IEEE International Conference on, pp. 455-462. IEEE (2004)

16. Erol-Kantarci, M., Mouftah, H.T.: Wireless multimedia sensor and actor networks for the next generation power grid. Ad Hoc Networks 9(4), 542-551 (2011)

17. Erol-Kantarci, M., Mouftah, H.T.: Wireless sensor networks for cost-efficient residential energy management in the smart grid. IEEE Trans. Smart Grid 2(2), 314-325 (2011)

18. Erol-Kantarci, M., Mouftah, H.T.: Smart grid forensic science: applications, challenges, and open issues. IEEE Communications Magazine 51(1), 68-74 (2013)

19. Erol-Kantarci, M., Mouftah, H.T.: Energy-efficient information and communication infrastructures in the smart grid: A survey on interactions and open issues. IEEE Communications Surveys and Tutorials 17(1), 179-197 (2015). DOI 10.1109/COMST.2014.2341600

20. Fadel, E.A., Gungor, V.C., Nassef, L., Akkari, N., Malik, M.G.A., Almasri, S., Akyildiz, I.F.: A survey on wireless sensor networks for smart grid. Computer Communications 71, 22-33 (2015). DOI 10.1016/j.comcom.2015.09.006

21. Fan, Z., Kalogridis, G., Efthymiou, C., Sooriyabandara, M., Serizawa, M., McGeehan, J.: The new frontier of communications research: Smart grid and smart metering. In: Proceedings of the 1st International Conference on Energy-Efficient Computing and Networking, e-Energy '10, pp. 115-118. ACM, New York, NY, USA (2010). DOI 10.1145/1791314.1791331

22. Fan, Z., Kulkarni, P., Gormus, S., Efthymiou, C., Kalogridis, G., Sooriyabandara, M., Zhu, Z., Lambotharan, S., Chin, W.H.: Smart grid communications: Overview of research challenges, solutions, and standardization activities. IEEE Communications Surveys and Tutorials 15(1), 21-38 (2013)

23. Fang, X., Misra, S., Xue, G., Yang, D.: Smart grid - the new and improved power grid: A survey. IEEE Communications Surveys and Tutorials 14(4), 944-980 (2012)

24. Fateh, B., Govindarasu, M., Ajjarapu, V.: Wireless network design for transmission line monitoring in smart grid. IEEE Trans. Smart Grid 4(2), 1076-1086 (2013)

25. Gao, J., Xiao, Y., Liu, J., Liang, W., Chen, C.L.P.: A survey of communication/networking in smart grids. Future Generation Comp. Syst. 28(2), 391-404 (2012)

26. Grilo, A., Casaca, A., Pereira, P.R., Buttyán, L., Gonçalves, J., Fortunato, C.: A wireless sensor and actuator network for improving the electrical power grid dependability. In: NGI, pp. 71-78 (2012)

27. Guglielmo, D.D., Brienza, S., Anastasi, G.: IEEE 802.15.4e: A survey. Computer Communications 88, 1-24 (2016). DOI 10.1016/j.comcom.2016.05.004

28. Gungor, V., Sahin, D.: Cognitive radio networks for smart grid applications: A promising technology to overcome spectrum inefficiency. Vehicular Technology Magazine, IEEE 7(2), 41-46 (2012). DOI 10.1109/MVT.2012.2190183

29. Gungor, V.C., Korkmaz, M.K.: Wireless link-quality estimation in smart grid environments. International Journal of Distributed Sensor Networks 2012 (2012)

30. Gungor, V.C., Lu, B., Hancke, G.P.: Opportunities and challenges of wireless sensor networks in smart grid. IEEE Transactions on Industrial Electronics 57(10), 3557-3564 (2010)

31. Gungor, V.C., Sahin, D., Kocak, T., Ergüt, S., Buccella, C., Cecati, C., Hancke, G.P.: Smart grid technologies: Communication technologies and standards. IEEE Trans. Industrial Informatics 7(4), 529-539 (2011)

32. Gungor, V.C., Sahin, D., Kocak, T., Ergüt, S., Buccella, C., Cecati, C., Hancke, G.P.: A survey on smart grid potential applications and communication requirements. IEEE Trans. Industrial Informatics 9(1), 28-42 (2013)

33. Henderson, T.R., Lacage, M., Riley, G.F., Dowell, C., Kopena, J.: Network simulations with the NS-3 simulator. SIGCOMM demonstration 15, 17 (2008)

34. Ho, Q., Gao, Y., Rajalingham, G., Le-Ngoc, T.: Wireless Communications Networks for the Smart Grid. Springer Briefs in Computer Science. Springer (2014). DOI 10.1007/9783-319-10347-1

35. std IEEE 802.15.4e: Part. 15.4: Low-rate wireless personal area networks (lr-wpans) amendment 1: Mac sublayer) (2012)

36. Incel, O.D.: A survey on multi-channel communication in wireless sensor networks. Computer Networks 55(13), 3081-3099 (2011)

37. Jaikaeo, C., Shen, C.C.: Qualnet tutorial. Retrieved Jan 6 (2005)

38. Khan, R.H., Khan, J.Y.: A comprehensive review of the application characteristics and traffic requirements of a smart grid communications network. Computer Networks $\mathbf{5 7}(3)$, 825 - 845 (2013). DOI http://dx.doi.org/10.1016/j.comnet.2012.11.002 
39. Levis, P., Lee, N., Welsh, M., Culler, D.: Tossim: Accurate and scalable simulation of entire tinyos applications. In: Proceedings of the 1st International Conference on Embedded Networked Sensor Systems, SenSys '03, pp. 126-137. ACM, New York, NY, USA (2003). DOI 10.1145/958491.958506

40. Liberatore, V., Al-Hammouri, A.: Smart grid communication and co-simulation. In: Energytech, 2011 IEEE, pp. 1-5 (2011). DOI 10.1109/EnergyTech.2011.5948542

41. Lin, H., Veda, S.S., Shukla, S.K., Mili, L., Thorp, J.S.: GECO: Global event-driven cosimulation framework for interconnected power system and communication network. IEEE Trans. Smart Grid 3(3), 1444-1456 (2012)

42. Long, X., Dong, M., Xu, W., Li, Y.W.: Online monitoring of substation grounding grid conditions using touch and step voltage sensors. IEEE Trans. Smart Grid 3(2), 761-769 (2012)

43. Ma, R., Chen, H.H., Huang, Y.R., Meng, W.: Smart grid communication: Its challenges and opportunities. Smart Grid, IEEE Transactions on 4(1), 36-46 (2013). DOI 10.1109/TSG.2012.2225851

44. Mahmood, A., Javaid, N., Razzaq, S.: A review of wireless communications for smart grid. Renewable and Sustainable Energy Reviews 41, 248-260 (2015)

45. Majumder, R., Bag, G., Kim, K.H.: Power sharing and control in distributed generation with wireless sensor networks. IEEE Trans. Smart Grid 3(2), 618-634 (2012)

46. Matta, N., Rahim-Amoud, R., Merghem-Boulahia, L., Jrad, A.: A wireless sensor network for substation monitoring and control in the smart grid. In: GreenCom, pp. 203-209 (2012)

47. Nasipuri, A., Cox, R., Conrad, J., der Zel, L.V., Rodriguez, B., McKosky, R.: Design considerations for a large-scale wireless sensor network for substation monitoring. In: LCN, pp. 866-873 (2010)

48. Osterlind, F., Dunkels, A., Eriksson, J., Finne, N., Voigt, T.: Cross-level sensor network simulation with cooja. In: Local Computer Networks, Proceedings 2006 31st IEEE Conference on, pp. 641-648 (2006). DOI 10.1109/LCN.2006.322172

49. Palattella, M., Accettura, N., Vilajosana, X., Watteyne, T., Grieco, L., Boggia, G., Dohler, M.: Standardized protocol stack for the internet of (important) things. Communications Surveys Tutorials, IEEE 15(3), 1389-1406 (2013). DOI 10.1109/SURV.2012.111412.00158

50. Rajalingham, G., Gao, Y., Ho, Q., Le-Ngoc, T.: Quality of service differentiation for smart grid neighbor area networks through multiple RPL instances. In: Q2SWinet'14, Proceedings of the 10th ACM Symposium on QoS and Security for Wireless and Mobile Networks, Montreal, QC, Canada, September 21-22, 2014, pp. 17-24 (2014). DOI 10.1145/2642687.2642695

51. Rekik, S., Baccour, N., Jmaiel, M., Drira, K.: Low-Power link quality estimation in smart grid environments. In: IWCMC 2015 Wireless Sensor Symposium (IWCMC 2015 Wireless Sensor Symposium). Dubrovnik, Croatia (2015)

52. Rekik, S., Baccour, N., Jmaiel, M., Drira, K.: Holistic link quality estimation-based routing metric for RPL networks in smart grids. In: 2016 IEEE 27th Annual IEEE International Symposium on Personal, Indoor and Mobile Radio Communications - (PIMRC): Mobile and Wireless Networks (IEEE PIMRC2016 Mobile and Wireless) (2016)

53. Sabbah, A.I., Mougy, A.H.E., Ibnkahla, M.: A survey of networking challenges and routing protocols in smart grids. IEEE Trans. Industrial Informatics 10(1), 210-221 (2014). DOI 10.1109/TII.2013.2258930

54. Sahin, D., Gungor, V.C., Kocak, T., Tuna, G.: Quality-of-service differentiation in singlepath and multi-path routing for wireless sensor network-based smart grid applications. Ad Hoc Networks 22, 43-60 (2014)

55. Shah, G., Gungor, V., Akan, O.: A cross-layer QoS-aware communication framework in cognitive radio sensor networks for smart grid applications. Industrial Informatics, IEEE Transactions on 9(3), 1477-1485 (2013). DOI 10.1109/TII.2013.2242083

56. Shah, G.A., Gungor, V.C., Akan, Ö.B.: A cross-layer design for QoS support in cognitive radio sensor networks for smart grid applications. In: ICC, pp. 1378-1382 (2012)

57. Sobeih, A., Hou, J.C., Kung, L., Li, N., Zhang, H., Chen, W., Tyan, H., Lim, H.: J-sim: a simulation and emulation environment for wireless sensor networks. IEEE Wireless Commun. 13(4), 104-119 (2006). DOI 10.1109/MWC.2006.1678171

58. Sobeih, A., Viswanathan, M., Marinov, D., Hou, J.C.: J-sim: An integrated environment for simulation and model checking of network protocols. In: IPDPS, pp. 1-6 (2007)

59. Soua, R., Minet, P.: Multichannel assignment protocols in wireless sensor networks: A comprehensive survey. Pervasive and Mobile Computing 16, 2-21 (2015). DOI 10.1016/j.pmcj.2014.04.004 
60. Sun, W., Wang, J.: Cross-layer QoS optimization of wireless sensor network for smart grid. International Journal of Distributed Sensor Networks (2014)

61. Temel, c., Gungor, V.c., Koçak, T.: Routing protocol design guidelines for smart grid environments. Comput. Netw. 60, 160-170 (2014). DOI 10.1016/j.bjp.2013.11.009

62. Varga, A., et al.: The omnet ++ discrete event simulation system. In: Proceedings of the European simulation multiconference (ESM2001), vol. 9, p. 65. sn (2001)

63. Wang, W., Xu, Y., Khanna, M.: A survey on the communication architectures in smart grid. Computer Networks 55(15), 3604-3629 (2011)

64. Wu, Y.C., Cheung, L.F., Lui, K.S., Pong, P.W.T.: Efficient communication of sensors monitoring overhead transmission lines. IEEE Trans. Smart Grid 3(3), 1130-1136 (2012)

65. Yan, Y., Qian, Y., Sharif, H., Tipper, D.: A survey on smart grid communication infrastructures: Motivations, requirements and challenges. IEEE Communications Surveys and Tutorials 15(1), 5-20 (2013)

66. Yigit, M., Gungor, V.C., Fadel, E.A., Nassef, L., Akkari, N., Akyildiz, I.F.: Channel-aware routing and priority-aware multi-channel scheduling for wsn-based smart grid applications. J. Network and Computer Applications 71, 50-58 (2016). DOI 10.1016/j.jnca.2016.05.015

67. Yigit, M., Incel, Ö.D., Çagri Güngör, V.: On the interdependency between multi-channel scheduling and tree-based routing for wsns in smart grid environments. Computer Networks 65, 1-20 (2014)

68. Yigit, M., Yoney, E., Gungor, V.: Performance of mac protocols for wireless sensor networks in harsh smart grid environment. In: Communications and Networking (BlackSeaCom), 2013 First International Black Sea Conference on, pp. 50-53 (2013). DOI 10.1109/BlackSeaCom.2013.6623380

69. Zaker, N., Kantarci, B., Erol-Kantarci, M., Mouftah, H.T.: Smart grid monitoring with service differentiation via EPON and wireless sensor network convergence. Optical Switching and Networking 14, 53-68 (2014). DOI 10.1016/j.osn.2014.01.010

70. Zhu, K., Chenine, M., Nordstrom, L.: ICT architecture impact on wide area monitoring and control systems' reliability. Power Delivery, IEEE Transactions on 26(4), 2801-2808 (2011). DOI 10.1109/TPWRD.2011.2160879 